\title{
New fuzzy spheres through confining potentials and energy cutoffs
}

\section{Gaetano Fiore*, Francesco Pisacane}

Dip. di Matematica e Applicazioni, Università di Napoli "Federico II", \& INFN, Sezione di Napoli,

Complesso Universitario M. S. Angelo, Via Cintia, 80126 Napoli, Italy

E-mail: gaetano.fiore@unina.it, francesco.pisacane@unina.it

We briefly report on our recent construction [1] of new fuzzy spheres $S_{\Lambda}^{d}$ of dimensions $d=1,2$ covariant under the full orthogonal group $O(D), D=d+1$. $S_{\Lambda}^{d}$ is built imposing a suitable energy cutoff on a quantum particle in $\mathbb{R}^{D}$ subject to a confining potential well $V(r)$ with a very sharp minimum on the sphere of radius $r=1$; the cutoff and the depth of the well depend on (and diverge with) $\Lambda \in \mathbb{N}$. The commutator of the coordinates depends only on the angular momentum, as in Snyder noncommutative spaces. As $\Lambda \rightarrow \infty$ the Hilbert space dimension diverges, $S_{\Lambda}^{d} \rightarrow S^{d}$, and we recover ordinary quantum mechanics on $S^{d}$. These models might be useful in quantum field theory, quantum gravity or condensed matter physics.

Corfu Summer Institute 2017 "School and Workshops on Elementary Particle Physics and Gravity", 2-28 September 2017, Corfu, Greece

\footnotetext{
${ }^{*}$ Speaker.
} 


\section{Introduction}

Nowadays noncommutative space(time) algebras are introduced and studied for several reasons. In particular: to regularize ultraviolet (UV) divergences in quantum field theory (QFT) [2]; as an arena to formulate Quantum Gravity $(\mathrm{QG})$ that naturally induces uncertainty relations of the type $\Delta x \gtrsim L_{p}$, as predicted by QG arguments (see e.g. [3, 4]); as an arena for the unification of fundamental interactions (see e.g. [5, 6]). Today Noncommutative Geometry [7, 8, 9, 10] is a sophisticated framework that develops the whole machinery of differential geometry on noncommutative spaces. Fuzzy spaces are particularly appealing noncommutative spaces: a fuzzy space is a sequence $\{\mathscr{A}\}_{n \in \mathbb{N}}$ of finite-dimensional algebras such that $\mathscr{A}_{n} \stackrel{n \rightarrow \infty}{\longrightarrow} \mathscr{A} \equiv$ algebra of regular functions on an ordinary manifold, with $\operatorname{dim}\left(\mathscr{A}_{n}\right) \stackrel{n \rightarrow \infty}{\longrightarrow} \infty$. The first and seminal fuzzy space is the Fuzzy Sphere (FS) of Madore and Hoppe [11, 12], the first applications to QFT models are in [13, 14]; $\mathscr{A}_{n} \simeq M_{n}(\mathbb{C})$ is generated by coordinates $x^{i}(i=1,2,3)$ fulfilling

$$
\left[x^{i}, x^{j}\right]=\frac{2 i}{\sqrt{n^{2}-1}} \varepsilon^{i j k} x^{k}, \quad r^{2}:=x^{i} x^{i}=1, \quad n \in \mathbb{N} \backslash\{1\}
$$

(sum over repeated indices is understood); in fact they are obtained by the rescaling $x^{i}=2 L_{i} / \sqrt{n^{2}-1}$ of the elements $L_{i}$ of the standard basis of so(3) in the irreducible representation $\left(\pi_{l}, V_{l}\right)$ characterized by $L_{i} L_{i}=l(l+1), n=2 l+1$. Fuzzy spaces have raised a big interest in the high energy physics community as a non-perturbative technique in QFT based on a finite-discretization of space(time) alternative to the lattice one: the main advantage is that the algebras $\mathscr{A}_{n}$ can carry representations of Lie groups (not only of discrete ones). They can be used also for internal (e.g. gauge) degrees of freedom (see e.g. [15]), or as a new tool in string and $D$-brane theories (see e.g. [16, 17]).

Relations (1.1) are covariant under $S O(3)$, but not under the whole $O(3)$; in particular not under parity $x^{i} \mapsto-x^{i}$, as the ordinary sphere $S^{2}$. In our opinion, another reason why the FS does not approximate $S^{2}$ in the best possible way is that $V_{l}$ carries an irreducible representation of $S O(3)$ (so that the Casimir $r^{2}$ is identically 1), whereas the Hilbert space of a quantum particle on $S^{2}$ has the following decomposition in irreducible representations of $S O(3)$ :

$$
\mathscr{L}^{2}\left(S^{2}\right)=\bigoplus_{l=0}^{\infty} V_{l} .
$$

Here we present new fuzzy approximations [1] of quantum mechanics (QM) on $S^{d}(d=1,2)$ overcoming these two problems: We start with an ordinary zero-spin quantum particle in $\mathbb{R}^{D}$ configuration space $(D=d+1)$ with Hamiltonian

$$
H=-\frac{1}{2} \Delta+V(r)
$$

Here $r^{2}:=x^{i} x^{i}, \Delta:=\partial_{i} \partial_{i}, \partial_{i} \equiv \partial / \partial x^{i}, i=1, \ldots, D$; we use dimensionless cartesian coordinates, momentum components and Hamiltonian $x^{i}, p_{i}:=-i \partial_{i}, H . x^{i}, p_{i}$ generate the Heisenberg algebra $\mathscr{O}$ of observables. The canonical commutation relations $\left[x^{i}, x^{j}\right]=0,\left[p_{i}, p_{j}\right]=0,\left[x^{i}, p_{j}\right]=i \delta_{j}^{i}$ as well as the Hamiltonian are invariant under all orthogonal transformations $x^{i} \mapsto x^{i}=Q_{j}^{i} x^{j}\left(Q^{-1}=\right.$ $Q^{T}$ ), including parity $Q=-I$. We choose $V(r)$ as a confining potential with a very sharp minimum 
at $r=1$, i.e. with $V^{\prime}(1)=0$ and very large $k:=V^{\prime \prime}(1) / 4>0$, and fix $V_{0}:=V(1)$ so that the ground state has zero energy, $E_{0}=0$. We choose an energy cutoff $\bar{E}$ satisfying first of all the condition

$$
V(r) \simeq V_{0}+2 k(r-1)^{2} \quad \text { if } r \text { fulfills } \quad V(r) \leq \bar{E},
$$

so that $V(r)$ is approximately harmonic in the classical region $v_{\bar{E}}$ compatible with the energy cutoff $V(r) \leq \bar{E}$. Then we project the theory onto the finite-dimensional Hilbert subspace $\mathscr{H}_{\bar{E}} \subset \mathscr{H} \equiv$ $\mathscr{L}^{2}\left(\mathbb{R}^{D}\right)$ spanned by $\psi$ fulfilling the eigenvalue equation

$$
H \psi=E \psi, \quad \psi \in \mathscr{L}^{2}\left(\mathbb{R}^{D}\right),
$$

with $E \leq \bar{E}$. This entails replacing every observable $A$ by $\bar{A}$ :

$$
A \mapsto \bar{A}:=P_{\bar{E}} A P_{\bar{E}},
$$

where $P_{\bar{E}}$ is the projection on $\mathscr{H}_{\bar{E}} . H, L_{i j}, P_{\bar{E}}$ commute; $L_{i j}:=x^{i} p_{j}-x^{j} p_{i}$ are the angular momentum components. Decomposing the Laplacian on $\mathbb{R}^{D}$ in polar coordinates $r, \varphi, \ldots$

$$
\Delta=\partial_{r}^{2}+(D-1) \frac{1}{r} \partial_{r}-\frac{1}{2 r^{2}} L_{i j} L_{i j}
$$

recalling that the eigenvalues of the square angular momentum $L^{2}=L_{i j} L_{i j} / 2$ are $j(j+D-2)$, and using the Ansatz $\psi=f(r) Y(\varphi, \ldots) \quad\left[Y\right.$ are eigenfunctions of $L^{2}$ and of the elements of a Cartan subalgebra of $s o(D)]$ we transform (1.5) into this auxiliary ODE in the unknown $f(r)$ :

$$
\left[-\partial_{r}^{2}-\frac{D-1}{r} \partial_{r}+\frac{j(j+D-2)}{r^{2}}+V(r)\right] f(r)=E f(r) .
$$

To obtain the lowest eigenvalues at leading order in $1 / k$ we don't need to solve it exactly: condition (1.4) allows us to approximate (1.7) with the eigenvalue equation of a 1-dimensional harmonic oscillator, by Taylor expanding $V(r), 1 / r, 1 / r^{2}$ around $r=1$.

As a second condition on the cutoff we ask that it be sufficiently low to 'freeze' radial excitations, so that the eigenvalues of $H$ fulfilling $E \leq \bar{E}$ coincide at leading order with those of the square angular momentum $L^{2}=L_{i j} L_{i j}$, i.e. with the Laplacian on the sphere $\mathbb{S}^{d}$; this can be considered as a quantum version of the constraint $r=1$. It turns out that on $\mathscr{H}_{\bar{E}}$ the $x^{i}$ are noncommutative à $l a$ Snyder, namely their commutators depend only on the angular momentum, and that they generate the whole algebra of observables. The whole procedure is $O(D)$-covariant by construction. Making $\bar{E}, V^{\prime \prime}(1) \gg 0$ diverge with some $\Lambda \in \mathbb{N}$ (while $E_{0}=0$ ), and keeping the leading terms in $1 / \Lambda$, we get a sequence $\{\mathscr{A}\}_{\Lambda \in \mathbb{N}}$ of fuzzy approximations of ordinary quantum mechanics (QM) on $S^{d}$. On $\mathscr{H}_{\bar{E}} \equiv \mathscr{H}_{\Lambda}=\bigoplus_{l=0}^{\Lambda} V_{l}$ the square distance $\mathscr{R}^{2}$ from the origin is not identically 1 , but a function of $L^{2}$, whose spectrum collapses to 1 in the $\Lambda \rightarrow \infty$ limit.

Our construction is inspired by the Landau model, where noncommuting $x, y$ are obtained projecting QM with a strong uniform magnetic field $B$ on the lowest energy subspace; therefore the method is physically sound. Our models might have applications to quantum models in condensed matter physics with an effective one- or two-dimensional configuration space in the form of a circle, a cylinder or a sphere, because they respect parity, and the restriction to the circle, cylinder or sphere 
is an effective one obtained "a posteriori" from the exact dynamics in the physical dimension 3. But we think that they are interesting also as new toy-models of fuzzy geometries in quantum field theory, quantum gravity, string theory. Our procedure can be generalized in a straightforward manner to $D>3$, as well as to other confining potentials; the dimension of the accessible Hilbert space $\mathscr{H}_{\bar{E}}$ will be approximately $\mathscr{B} / h^{D}$, where $h, \mathscr{B}$ are the Planck constant and the volume of the classically allowed region in phase space (i.e. the one characterized by energies $E \leq \bar{E}$ ). If $H$ is invariant under some symmetry group, then the projection $P_{\bar{E}}$ on $\mathscr{H}_{\bar{E}}$ is invariant as well, and the projected theory will inherit that symmetry. Imposing a cutoff $\bar{E}$ on a given theory may have various motivations, in particular: it can yield an effective description of a system when our preparation of the system, or our measurements, or the interactions with the environment, cannot bring its state to energies $E>\bar{E}$; or it may even be a necessity if we believe $\bar{E}$ represents the threshold for the onset of new physics not accountable by that theory.

In sections 2, 3 we treat the cases $D=2,3$ leading to $S_{\Lambda}^{1}, S_{\Lambda}^{2}$ respectively. Section 4 contains a comparison with the literature, final remarks, outlook and conclusions. For more details, explicit computations and proofs we refer the reader to Ref. [1].

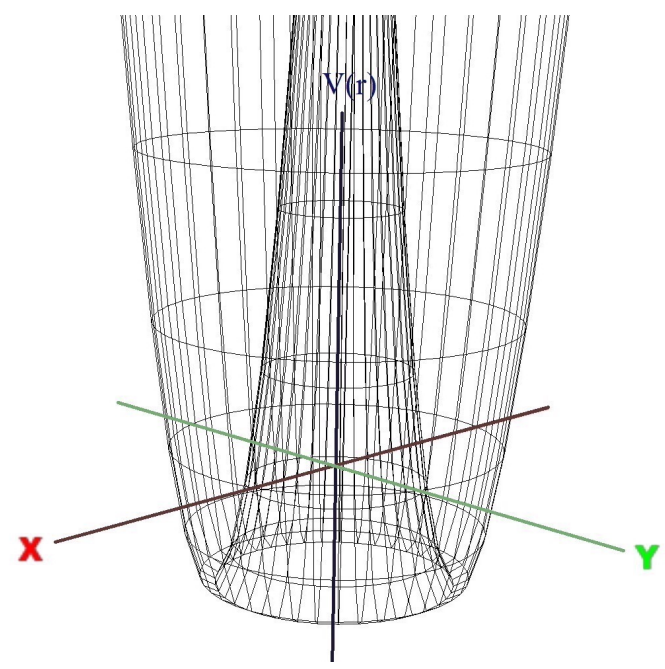

Figure 1: Three-dimensional plot of $V(r)$

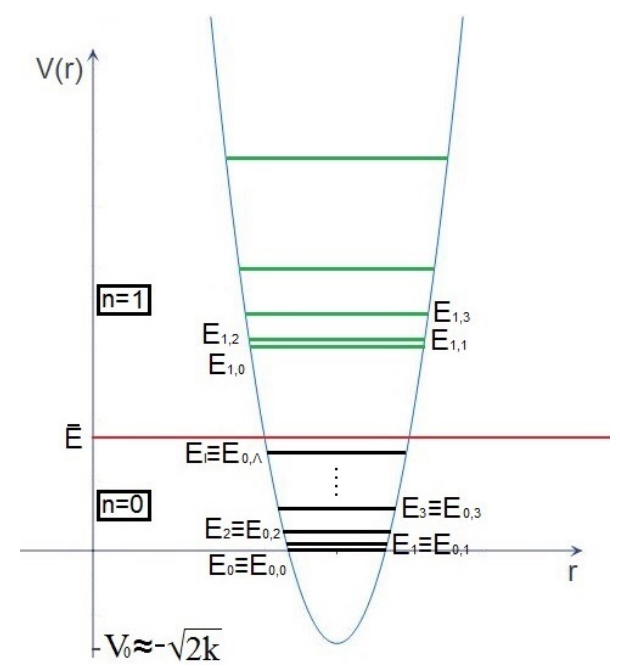

Figure 2: Two-dimensional plot of $V(r)$ including the energy-cutoff

\section{2. $D=2: O(2)$-covariant fuzzy circle}

The potential is shown in fig. 1. For convenience we look for the solution $\psi$ of (1.5) in the form $\psi=e^{i m \varphi} f(\rho)$, with $m \in \mathbb{Z} \equiv$ spectrum of $L \equiv L_{12}, \rho:=\ln r$, and expand around $\rho=0$. The harmonic oscillator approximation of (1.7) has eigenvalues and (Hérmite) eigenfunctions

$$
\begin{aligned}
& E=E_{n, m}=2 n \sqrt{2 k}-2 n+m^{2}+O(1 / \sqrt{k}) \\
& f_{n, m}(\rho)=N_{n, m} \exp \left[-\frac{\left(\rho-\widetilde{\rho}_{n, m}\right)^{2} \sqrt{k_{n, m}}}{2}\right] H_{n}\left[\left(\rho-\widetilde{\rho}_{n, m}\right) \sqrt[4]{k_{n, m}}\right], \\
& k_{n, m}=2\left(k-E_{n, m}+V_{0}\right), \quad \widetilde{\rho}_{n, m}=\frac{E_{n, m}-V_{0}}{k_{n, m}},
\end{aligned}
$$


with $n \in \mathbb{N}_{0}$; we have set $V_{0}=-\sqrt{2 k}+2+O\left(\frac{1}{\sqrt{k}}\right)$ to fulfill the requirement $E_{0,0}=0$. Up to terms $O\left(\frac{1}{\sqrt{k}}\right)(2.1)$ gives

$$
E_{m} \equiv E_{0, m}=m^{2}
$$

which are the eigenvalues of the Laplacian $L^{2}$ on $S^{1}$, while $E_{n, m} \rightarrow \infty$ as $k \rightarrow \infty$ if $n>0$ we have (we have highlighted the unwelcome $n$-dependent contribution in red, the welcome $m$-dependent one in blue); we can eliminate the latter eigenvalues by choosing a cutoff $\bar{E}<2 \sqrt{2 k}-2$. The eigenfunctions of $H$ corresponding to $E=E_{m}$ are

$$
\psi_{m}(\rho, \varphi)=N_{m} e^{i m \varphi} e^{-\frac{\left(\rho-\tilde{\rho}_{m}\right)^{2} \sqrt{k_{m}}}{2}} .
$$

We set $\Lambda:=[\sqrt{\bar{E}}] \in \mathbb{N}$ and abbreviate $\mathscr{H}_{\Lambda} \equiv \mathscr{H}_{\bar{E}} . E_{m} \leq \bar{E}$ implies

$$
m^{2} \leq \Lambda^{2}<2 \sqrt{2 k}-2
$$

so that all $E_{m}$ are smaller than the energy levels corresponding to $n>0$ (see fig. 2). Clearly $\operatorname{dim}\left(\mathscr{H}_{\Lambda}\right)=2 \Lambda+1$. We recover the whole spectrum of $L^{2}$ on $S^{1}$ by allowing $\sqrt{\bar{E}}$, or equivalently $\Lambda$, to diverge with $k$ while respecting (2.4).

Let $x^{ \pm}:=\frac{x \pm i y}{\sqrt{2}}=r e^{ \pm i \varphi}$. By explicit computations

$$
\left\langle\psi_{n}, x^{ \pm} \psi_{m}\right\rangle=\frac{a}{\sqrt{2}}\left[1+\frac{m(m \pm 1)}{2 k}\right] \delta_{m \pm 1}^{n}
$$

with $a=1+\frac{9}{4} \frac{1}{\sqrt{2 k}}+\frac{137}{64 k}+\ldots$ To get rid of $a$ we rescale $\xi^{ \pm}:=\frac{\bar{x}^{ \pm}}{a} . \bar{x}^{-}, \xi^{-}$are resp. the adjoints of $\bar{x}^{+}, \xi^{+}$. Then, up to terms $O\left(1 / k^{3 / 2}\right)$

$$
\begin{aligned}
& \xi^{ \pm} \psi_{m}= \begin{cases}\frac{1}{\sqrt{2}}\left[1+\frac{m(m \pm 1)}{2 k}\right] \psi_{m \pm 1} & \text { if }-\Lambda \leq \pm m \leq \Lambda-1 \\
0 & \text { otherwise, }\end{cases} \\
& \bar{L} \psi_{m}=m \psi_{m} .
\end{aligned}
$$

We define the square distance from the origin as the $O(2)$-invariant $\mathscr{R}^{2}:=\xi^{+} \xi^{-}+\xi^{-} \xi^{+}$; let $\widetilde{P}_{m}$ be the projection over the 1 -dim subspace spanned by $\psi_{m}$. Eq. (2.6) implies at leading order in $1 / k$

$$
\begin{gathered}
{\left[\xi^{+}, \xi^{-}\right]=-\underbrace{\frac{\bar{L}}{k}}_{\text {Snyder-like }}+\left[1+\frac{\Lambda(\Lambda+1)}{k}\right] \frac{\widetilde{P}_{\Lambda}-\widetilde{P}_{-\Lambda}}{2} .} \\
\prod_{m=-\Lambda}^{\Lambda}(\bar{L}-m I)=0, \quad(\bar{L})^{\dagger}=\bar{L}, \\
{\left[\bar{L}, \xi^{ \pm}\right]= \pm \xi^{ \pm}, \quad \xi^{+\dagger}=\xi^{-}, \quad\left(\xi^{ \pm}\right)^{2 \Lambda+1}=0 .} \\
\mathscr{R}^{2}=1+\frac{\bar{L}^{2}}{k}-\left[1+\frac{\Lambda(\Lambda+1)}{k}\right] \frac{\widetilde{P}_{\Lambda}+\widetilde{P}_{-\Lambda}}{2} .
\end{gathered}
$$


Eq. (2.7-2.10) are exact if we adopt (2.6) as definitions of $\xi^{+}, \xi^{-}, \bar{L}$. To obtain a fuzzy space we can choose $k$ as a function of $\Lambda$ fulfilling (2.4), for example $k=\Lambda^{2}(\Lambda+1)^{2}$, and the commutative limit will be $\Lambda \rightarrow \infty$. Then e.g. (2.7) becomes

$$
\left[\xi^{+}, \xi^{-}\right]=\frac{-\bar{L}}{\Lambda^{2}(\Lambda+1)^{2}}+\left[1+\frac{1}{\Lambda(\Lambda+1)}\right] \frac{\widetilde{P}_{\Lambda}-\widetilde{P}_{-\Lambda}}{2} .
$$

Let us summarize what we have found so far:

- The matched confining potential and energy cutoff lead to a non-zero commutator of the coordinates of the Snyder's Lie algebra type, i.e. depending only on $L$, and vanishing as $k \rightarrow \infty$. To obtain a fuzzy space we can choose $k$ as a function of $\Lambda$ fulfilling (2.4); one possible choice is $k=\Lambda^{2}(\Lambda+1)^{2}$, and the commutative limit will be $\Lambda \rightarrow+\infty$.

- $\mathscr{R}^{2} \neq 1$; but it is a function of $L^{2}$, and, for each fixed $\Lambda$, its eigenvalues (except the highest one) are close to 1 , slightly grow with $m$ and collapse to 1 as $\Lambda \rightarrow \infty$.

- Relations (2.7-2.10) are $O(2)$-invariant, because in the original model both the commutation relations and $H$ (hence also $P_{\bar{E}}$ ) are.

- The ordered monomials $\left(\xi^{+}\right)^{h}(\bar{L})^{l}\left(\xi^{-}\right)^{n}$ [with degrees $h, l, n$ bounded by (2.8-2.9)] make up a basis of the $(2 \Lambda+1)^{2}$-dim vector space $\mathscr{A}_{\Lambda}:=\operatorname{End}\left(\mathscr{H}_{\Lambda}\right)$ (the $\widetilde{P}_{m}$ themselves can be expressed as polynomials in $\bar{L}$ ).

- $\xi^{+}, \xi^{-}$(or equivalently $\bar{x}^{+}, \bar{x}^{-}$) generate the $*$-algebra $\mathscr{A}_{\Lambda}$, because also $\bar{L}$ can be expressed as a non-ordered polynomial in $\xi^{+}, \xi^{-}$. An alternative set of generators is $\left\{E^{+}, E^{-}\right\}$in the $(2 \Lambda+1)$-dimensional representation of $s u(2)$ (see below).

- As $\Lambda \rightarrow \infty\left[\xi^{+}, \xi^{-}\right] \rightarrow 0, \operatorname{dim}\left(\mathscr{H}_{\Lambda}\right) \rightarrow \infty, \psi_{m} \rightarrow \delta(\rho) e^{i m \varphi}$.

What about the operators $\bar{\partial}_{ \pm}$? As seen, they are not needed as generators of $\mathscr{A}_{\Lambda}$. Actually, $\bar{\partial}_{ \pm}$do not go to $\partial_{ \pm}$as $\Lambda \rightarrow \infty$ because every $\partial_{ \pm} \psi_{m}$ has a non-negligible $n=1$ component. On the contrary, $\bar{L} \rightarrow L$; this is welcome, because in the limit $\Lambda \rightarrow \infty$ all vector fields tangential to $S^{1}$ are of the form $f(\varphi) L$.

\subsection{Realization of the algebra of observables through $U$ so (3)}

The algebra of observables $\mathscr{A}_{\Lambda}:=\operatorname{End}\left(\mathscr{H}_{\Lambda}\right)$ is isomorphic to

$$
\mathscr{A}_{\Lambda} \simeq M_{N}(\mathbb{C}) \simeq \pi_{\Lambda}[U s o(3)], \quad N=2 \Lambda+1,
$$

where $\pi_{\Lambda}$ is the $N$-dimensional unitary representation of $U s o(3)$. The latter is characterized by the condition $\pi_{\Lambda}(C)=\Lambda(\Lambda+1)$, where $C=E^{a} E^{-a}$ is the Casimir, and $E^{a}(a \in\{+, 0,-\})$ make up the Cartan-Weyl basis $E^{a}$ of $s o(3)$,

$$
\left[E^{+}, E^{-}\right]=E^{0}, \quad\left[E^{0}, E^{ \pm}\right]= \pm E^{ \pm}, \quad E^{a \dagger}=E^{-a} .
$$

To simplify the notation we drop $\pi_{\Lambda}$. In fact we can realize $\xi^{+}, \bar{L}, \xi^{-}$by setting

$$
\begin{gathered}
\bar{L}=E^{0}, \quad \bar{\xi}^{ \pm}=f_{ \pm}\left(E^{0}\right) E^{ \pm} \\
f_{+}(s)=\frac{1}{\sqrt{2}} \sqrt{\frac{1+s(s-1) / k}{\Lambda(\Lambda+1)-s(s-1)}}=f_{-}(s-1) .
\end{gathered}
$$




\section{$2.2 *$-Automorphisms of the algebra of observables}

Within the group $S U(N)$ of $*$-automorphisms of $M_{N}(\mathbb{C}) \simeq \mathscr{A}_{\Lambda}$

$$
a \mapsto g a g^{-1}, \quad a \in \mathscr{A}_{\Lambda} \simeq M_{N}, \quad g \in S U(N),
$$

a special role is played by the subgroup $S O(3)$ acting through the representation $\pi_{\Lambda}$, namely $g=$ $\pi_{\Lambda}\left[e^{i \alpha}\right]$, where $\alpha \in \operatorname{so}(3)$ is a combination with real coefficients of $E^{0}, E^{+}+E^{-}, i\left(E^{-}-E^{+}\right)$. $O(2) \subset S O(3)$ plays the role of isometry group. In particular, choosing $\alpha=\theta E^{0}$ amounts to a rotation by angle $\theta$ in the $\bar{x}^{1} \bar{x}^{2}$ plane: $\bar{L} \mapsto \bar{L}$ and

$$
\bar{x}^{ \pm} \mapsto \bar{x}^{\prime \pm}=e^{ \pm i \theta} \bar{x}^{ \pm} \quad \Leftrightarrow \quad\left\{\begin{array}{l}
\bar{x}^{\prime 1}=\bar{x}^{1} \cos \theta+\bar{x}^{2} \sin \theta \\
\bar{x}^{\prime 2}=-\bar{x}^{1} \sin \theta+\bar{x}^{2} \cos \theta
\end{array} .\right.
$$

Choosing $\alpha=\pi\left(E^{+}+E^{-}\right) / \sqrt{2}$ we obtain a $O(2)$-transformation with determinant $=-1$ in such a plane: $E^{0} \mapsto-E^{0}, E^{ \pm} \mapsto E^{\mp}$. As $f_{ \pm}(-s)=f_{ \pm}(1+s)=f_{\mp}(s)$, this is equivalent to $\bar{x}^{1} \mapsto \bar{x}^{1}$, $\bar{x}^{2} \mapsto-\bar{x}^{2}, \bar{L} \mapsto-\bar{L}$.

\subsection{Convergence to $O(2)$-covariant quantum mechanics on $S$ as $\Lambda \rightarrow \infty$}

Define the natural $O(2)$-covariant embedding $\mathscr{I}: \mathscr{H}_{\Lambda} \hookrightarrow \mathscr{L}^{2}(S) \equiv \mathscr{H}_{s}$ by setting $\mathscr{I}\left(\psi_{m}\right):=$ $u^{m}\left(u \equiv e^{i \varphi}\right)$ and applying linear extension; below we drop $\mathscr{I}$ and identify $\psi_{m}=u^{m}$ as elements of the Hilbert space. Clearly $P_{\Lambda} \phi \rightarrow \phi$ in the $\mathscr{H}_{s}$-norm \|\| , for all $\phi \in \mathscr{H}_{s}: \mathscr{H}_{\Lambda}$ 'invades' $\mathscr{H}_{s}$ as $\Lambda \rightarrow \infty$.

$\mathscr{I}$ induces an embedding $\mathscr{J}: \mathscr{A}_{\Lambda} \hookrightarrow B\left[\mathscr{H}_{S}\right]$ in the operator algebra, with $\mathscr{A}_{\Lambda}$ annihilating $\mathscr{H}_{\Lambda}^{\perp}$; one easily finds that $\bar{L}=L$ on $\mathscr{H}_{\Lambda}$, and $\bar{L} \phi \rightarrow L \phi$ as $\Lambda \rightarrow \infty$, for all $\phi \in D(L) \subset \mathscr{H}_{s}$. Bounded (resp. continuous) functions $f$ on $S$, acting as multiplication operators $f \cdot: \phi \in \mathscr{H}_{s} \mapsto f \phi \in \mathscr{H}_{s}$, make up a subalgebra $B(S)$ [resp. $C(S)]$ of $B\left[\mathscr{H}_{s}\right]$. The fuzzy analog of the vector space $B(S)$ is:

$$
\mathscr{C}_{\Lambda}:=\left\{\sum_{h=-2 \Lambda}^{2 \Lambda} f_{h} \eta^{h}, f_{h} \in \mathbb{C}\right\}
$$

where $\eta^{m} \equiv\left(\sqrt{2} \xi^{+}\right)^{m}, \eta^{-m} \equiv\left(\sqrt{2} \xi^{-}\right)^{m}, m \geq 0 . \mathscr{C}_{\Lambda} \subset \mathscr{A}_{\Lambda}$ as a vector space, but not as a subalgebra. One easily shows that $\eta^{h} \phi \rightarrow u^{h} \phi$. Moreover, setting $\hat{f}_{\Lambda}:=\sum_{h=-2 \Lambda}^{2 \Lambda} f_{h} \eta^{h} \in \mathscr{A}_{\Lambda}$ for all $f \in B(S)$, we find

Proposition 3.3 in [1]. Choose $k(\Lambda) \geq 2 \Lambda(\Lambda+1)(2 \Lambda+1)^{2}$. Then $\hat{f}_{\Lambda} \rightarrow f \cdot \widehat{(f g)_{\Lambda}} \rightarrow f g$. $\hat{f}_{\Lambda} \hat{g}_{\Lambda} \rightarrow f g$. strongly as $\Lambda \rightarrow \infty, \forall f, g \in B(S)$.

On the other hand, the corresponding convergences in the operator norm do not hold, because for all $\Lambda>0$ the operators $\bar{x}^{ \pm}, \bar{L}$ annihilate $\mathscr{H}_{\Lambda}^{\perp}$, whereas $u^{ \pm 1}, L$ do not.

\section{3. $\mathbf{D}=3: O(3)$-covariant fuzzy sphere}

We associate the pseudovector $L_{i}=\frac{1}{2} \varepsilon^{i j k} L_{j k}$ to the antisymmetric matrix $L_{i j}$ of the angular momentum components. For all vectors $\mathbf{v}$ depending on $\mathbf{x}, i \nabla$ we shall use either the components 
$v^{i}(i \in\{1,2,3\})$ or the ones $v^{a}(a \in\{-, 0,+\})$ defined by

$$
\left(\begin{array}{c}
v^{+} \\
v^{-} \\
v^{0}
\end{array}\right)=\underbrace{\left(\begin{array}{ccc}
\frac{1}{\sqrt{2}} & \frac{i}{\sqrt{2}} & 0 \\
\frac{1}{\sqrt{2}} & \frac{-i}{\sqrt{2}} & 0 \\
0 & 0 & 1
\end{array}\right)}_{U}\left(\begin{array}{c}
v^{1} \\
v^{2} \\
v^{3}
\end{array}\right) .
$$

( $U$ is a unitary matrix) which fulfill

$$
\left[L_{a}, v^{a}\right]=0, \quad\left[L_{0}, v^{ \pm}\right]= \pm v^{ \pm}, \quad\left[L_{ \pm}, v^{\mp}\right]= \pm v^{0}, \quad\left[L_{ \pm}, v^{0}\right]=\mp v^{ \pm} .
$$

In particular, $x^{0} \equiv z, x^{ \pm}=\frac{x^{1} \pm i x^{2}}{\sqrt{2}} \equiv \frac{x \pm i y}{\sqrt{2}}=\frac{r \sin \theta e^{ \pm i \varphi}}{\sqrt{2}}$. We make the Ansatz $\psi=\frac{f(r)}{r} Y_{l}^{m}(\theta, \varphi) . \quad Y_{l}^{m}$ are the spherical harmonics:

$$
L^{2} Y_{l}^{m}(\theta, \varphi)=l(l+1) Y_{l}^{m}(\theta, \varphi), \quad L_{3} Y_{l}^{m}(\theta, \varphi)=m Y_{l}^{m}(\theta, \varphi),
$$

with $l \in \mathbb{N}_{0}, m \in \mathbb{Z},|m| \leq l$. Under assumption (1.4) the harmonic oscillator approximation of (1.7) admits the (Hérmite) eigenfunctions

$$
f_{n, l}(r)=N_{n, l} e^{-\frac{\left(r-\widetilde{r}_{l}\right)^{2} \sqrt{k_{l}}}{2}} H_{n}\left(\left(r-\widetilde{r}_{l}\right) \sqrt[4]{k_{l}}\right), \quad n=0,1, \ldots
$$

with $k_{l}:=2 k+3 l(l+1), \widetilde{r}_{l}=\frac{2 k+4 l(l+1)}{2 k+3 l(l+1)}$. We set $V_{0}=-\sqrt{2 k}$ to fulfill the requirement $E_{0,0}=0$; then the energies associated to $\psi_{n, l, m}=\frac{f_{n, l}(r)}{r} Y_{l}^{m}(\theta, \varphi)$ are

$$
E_{n, l}=2 n \sqrt{2 k}+l(l+1)+O(1 / \sqrt{2 k}) .
$$

Again $E_{0, l}=l(l+1)=: E_{l}$ are the eigenvalues of the Laplacian $L^{2}$ on $S^{2}$, while $E_{n, l} \rightarrow \infty$ as $k \rightarrow \infty$ if $n>0$ (we have highlighted the unwelcome $n$-dependent contribution in red, the welcome $l$ dependent one in blue). We can eliminate the latter (i.e. constrain $n=0$ ) imposing a cutoff

$$
E \leq \Lambda(\Lambda+1) \equiv \bar{E}<2 \sqrt{2 k},
$$

namely projecting the theory on the Hilbert subspace $\mathscr{H}_{\Lambda} \subset \mathscr{L}^{2}\left(\mathbb{R}^{3}\right)$ spanned by

$$
\psi_{l}^{m}:=\psi_{0, l, m} \simeq \frac{N_{l}}{r} e^{-\frac{\left(r-\tilde{r}_{l}\right)^{2} \sqrt{k_{l}}}{2}} Y_{l}^{m}(\theta, \varphi), \quad|m| \leq l, \quad l \leq \Lambda .
$$

Clearly $\operatorname{dim}\left(\mathscr{H}_{\Lambda}\right)=(\Lambda+1)^{2}$. Multiplication by $x^{a}=r \frac{x^{a}}{r} \quad(a=-, 0,+)$ on $\psi_{l}^{m}$ factorizes into the one by $r$ on $\frac{f_{0, l}(r)}{r}$ and the one by $\frac{x^{a}}{r}$ on $Y_{l}^{m}$. After projection we find

$$
\begin{aligned}
& \bar{x}^{a} \psi_{l}^{m}=c_{l} A_{l}^{a, m} \psi_{l-1}^{m+a}+c_{l+1} A_{l+1}^{-a, m+a} \psi_{l+1}^{m+a}, \\
& c_{0}=c_{\Lambda+1}=0, \quad c_{l}=\sqrt{1+\frac{l^{2}}{k}} \quad 1 \leq l \leq \Lambda
\end{aligned}
$$

up to terms $O\left(1 / k^{\frac{3}{2}}\right)$; here $A_{l}^{a, m}, B_{l}^{a, m}$ are the coefficients determined by the ordinary multiplication rules

$$
\frac{x^{a}}{r} Y_{l}^{m}=A_{l}^{a, m} Y_{l-1}^{m+a}+B_{l}^{a, m} Y_{l+1}^{m+a},
$$


namely $B_{l}^{a, m}=A_{l+1}^{-a, m+a}$, and

$$
A_{l}^{ \pm, m}= \pm \sqrt{\frac{(l \mp m)(l \mp m-1)}{2(2 l+1)(2 l-1)}}, \quad A_{l}^{0, m}=\sqrt{\frac{(l+m)(l-m)}{(2 l+1)(2 l-1)}} .
$$

At leading order the $\bar{L}_{i}, \bar{x}^{i}, i \in\{1,2,3\}$, fulfill

$$
\begin{aligned}
& \prod_{l=0}^{\Lambda}\left[\bar{L}^{2}-l(l+1) I\right]=0, \quad \prod_{m=-l}^{l}\left(\bar{L}_{3}-m I\right) \widetilde{P}_{l}=0, \\
& \bar{L}_{i}^{\dagger}=\bar{L}_{i}, \quad\left[\bar{L}_{i}, \bar{L}_{j}\right]=i \varepsilon^{i j h} \bar{L}_{h}, \quad \bar{x}^{i \dagger}=\bar{x}^{i}, \quad \bar{x}^{i} \bar{L}_{i}=0, \\
& \underbrace{\left[\bar{L}_{i}, \bar{x}^{j}\right]=i \varepsilon^{i j h} \bar{x}^{h}}_{\text {Snyder-like }}, \quad\left[\bar{x}^{i}, \bar{x}^{j}\right]=i \varepsilon^{i j h} \underbrace{\left(-\frac{1}{k}+K \widetilde{P}_{\Lambda}\right) \bar{L}_{h}}_{\text {Snyder-like }},
\end{aligned}
$$

where $K=\frac{1}{k}+\frac{1+\frac{\Lambda^{2}}{k}}{2 \Lambda+1}, \bar{L}^{2}:=\bar{L}_{i} \bar{L}_{i}=\bar{L}_{a} \bar{L}_{-a}$ is $L^{2}$ projected on $\mathscr{H}_{\Lambda}$, and $\widetilde{P}_{l}$ is the projection on its eigenspace with eigenvalue $l(l+1)$. Moreover, the square distance from the origin is the $O(3)$ invariant

$$
\mathscr{R}^{2}:=\bar{x}^{i} \bar{x}^{i}=1+\frac{\bar{L}^{2}+1}{k}-\left[1+\frac{(\Lambda+1)^{2}}{k}\right] \frac{\Lambda+1}{2 \Lambda+1} \widetilde{P}_{\Lambda} .
$$

Relations (3.7-3.10) are exact if we adopt (3.5) as definitions of $\bar{x}^{a}$. To obtain a fuzzy space we can choose $k$ as a function of $\Lambda$ fulfilling (3.3); one possible choice is $k=\Lambda^{2}(\Lambda+1)^{2}$, and the commutative limit will be $\Lambda \rightarrow+\infty$. Again:

- The commutators $\left[\bar{x}^{i}, \bar{x}^{j}\right]$ (3.9) depend only on the angular momentum and are Snyder-like, i.e. (apart from the additional term in the second formula) are proportional to angular momentum components, and vanish as $\Lambda \rightarrow \infty$; in the same limit $\psi_{l}^{m} \rightarrow \delta(r-1) Y_{l}^{m}$.

- Hence (3.7-3.9) are covariant under the whole group $O(3)$, including parity $\bar{x}_{i} \mapsto-\bar{x}_{i}, \bar{L}_{i} \mapsto \bar{L}_{i}$, contrary to Madore's and Hoppe's FS.

- $\mathscr{R}^{2} \neq 1$; but it is a function of $L^{2}$, and, for each fixed $\Lambda$, its eigenvalues (except the highest one) are close to 1 , slightly grow with $l$ and collapse to 1 as $\Lambda \rightarrow \infty$.

- The ordered monomials in $\overline{x_{i}}, \overline{L_{i}}$ make up a basis of the $(\Lambda+1)^{4}$-dim vector space $\mathscr{A}:=$ $\operatorname{End}\left(\mathscr{H}_{\Lambda}\right) \simeq M_{(\Lambda+1)^{2}}(\mathbb{C})$, because the $\widetilde{P}_{l}$ themselves can be expressed as polynomials in $\bar{L}^{2}$.

- Actually, $\bar{x}_{i}$ generate the $*$-algebra $\mathscr{A}$, because also the $\bar{L}_{i}$ can be expressed as a non-ordered polynomial in the $\bar{x}_{i}$.

\subsection{Realization of the algebra $\mathscr{A}_{\Lambda}$ of observables through $U s o(4)$}

We recall that $s o(4) \simeq s u(2) \oplus s u(2) ;$ hence this Lie algebra is spanned by $\left\{E_{i}^{1}, E_{i}^{2}\right\}_{i=1}^{3}$ fulfilling

$$
\left[E_{i}^{1}, E_{j}^{2}\right]=0, \quad\left[E_{i}^{1}, E_{j}^{1}\right]=i \varepsilon^{i j k} E_{k}^{1}, \quad\left[E_{i}^{2}, E_{j}^{2}\right]=i \varepsilon^{i j k} E_{k}^{2}
$$


$L_{i}:=E_{i}^{1}+E_{i}^{2}, X_{i}:=E_{i}^{1}-E_{i}^{2}$ make up alternative basis of $s o(4)$ and fulfill

$$
\left[L_{i}, L_{j}\right]=i \varepsilon^{i j k} L_{k}, \quad\left[L_{i}, X_{j}\right]=i \varepsilon^{i j k} X_{k}, \quad\left[X_{i}, X_{j}\right]=i \varepsilon^{i j k} L_{k} .
$$

The $L_{i}$ close another $s u(2)$. Passing to generators labelled by $a \in\{-, 0,+\}$, we find

$$
\begin{gathered}
{\left[L_{+}, L_{-}\right]=L_{0}, \quad\left[L_{0}, L_{ \pm}\right]= \pm L_{ \pm}=\left[X_{0}, X_{ \pm}\right], \quad\left[X_{+}, X_{-}\right]=L_{0},} \\
{\left[L_{ \pm}, X_{\mp}\right]= \pm X_{0}, \quad\left[L_{0}, X_{ \pm}\right]= \pm X_{ \pm}=\left[X_{0}, L_{ \pm}\right], \quad\left[L_{a}, X_{a}\right]=0}
\end{gathered}
$$

(in the last formula there is no sum over $a$ ), where $L^{2}:=L_{i} L_{i}=L_{a} L_{-a}, X^{2}:=X_{i} X_{i}=X_{a} X_{-a}$.

In the representation $\pi_{\Lambda}:=\pi_{\frac{\Lambda}{2}} \otimes \pi_{\frac{\Lambda}{2}}$ of $U s o(4) \simeq U s u(2) \otimes U s u(2)$ on the Hilbert space $\mathbf{V}_{\Lambda}:=$ $V_{\frac{\Lambda}{2}} \otimes V_{\frac{\Lambda}{2}}$ it is $C^{1}:=E_{i}^{1} E_{i}^{1}=\frac{\Lambda}{2}\left(\frac{\Lambda}{2}+1\right)=E_{i}^{2} E_{i}^{2}=: C^{2}$, or equivalently

$$
X \cdot L=L \cdot X=0, \quad X^{2}+L^{2}=\Lambda(\Lambda+2)
$$

(we have dropped the symbols $\boldsymbol{\pi}_{\Lambda}$ ). $\mathbf{V}_{\Lambda}$ admits an orthonormal basis consisting of common eigenvectors of $L^{2}$ and $L_{0}$; in standard ket notation,

$$
L_{0}|l, m\rangle=m|l, m\rangle, \quad L^{2}|l, m\rangle=l(l+1)|l, m\rangle
$$

with $0 \leq l \leq \Lambda$ and $|m| \leq l . \quad \mathbf{V}_{\Lambda}, \mathscr{H}_{\Lambda}$ have the same dimension $(\Lambda+1)^{2}$ and decomposition in irreducible representations of the $L_{i}$ subalgebra; we identify them setting $\psi_{l}^{m} \equiv|l, m\rangle$. The action of $X^{a}$ on $\mathbf{V}_{\Lambda}$ reads

$$
\begin{aligned}
& X^{a}|l, m\rangle=d_{l} A_{l}^{a, m}|l-1, m+a\rangle+d_{l+1} B_{l}^{a, m}|l+1, m+a\rangle \\
& d_{l}:=\sqrt{(\Lambda+1)^{2}-l^{2}}
\end{aligned}
$$

We can naturally realize $\bar{L}_{a}, \bar{x}^{a}$ within $\pi_{\Lambda}[U s u(2) \otimes U s u(2)]$ [1]. Define $\lambda:=\frac{\sqrt{4 L^{2}+1}-1}{2}$; then $\lambda|l, m\rangle=l|l, m\rangle$. The Ansatz

$$
\bar{L}_{a}=L_{a}, \quad \bar{x}^{a}=g(\lambda) X^{a} g(\lambda),
$$

fulfills (3.5) and therefore (3.7-3.9), provided

$$
\begin{aligned}
g(l) & =\sqrt{\frac{\prod_{h=0}^{l-1}(\Lambda+l-2 h)}{\prod_{h=0}^{l}(\Lambda+l+1-2 h)} \prod_{j=0}^{\left[\frac{l-1}{2}\right]} \frac{1+\frac{(l-2 j)^{2}}{k}}{1+\frac{(l-1-2 j)^{2}}{k}}} \\
& =\sqrt{\frac{\Gamma\left(\frac{\Lambda+l}{2}+1\right) \Gamma\left(\frac{\Lambda-l+1}{2}\right)}{\Gamma\left(\frac{\Lambda+1+l}{2}+1\right) \Gamma\left(\frac{\Lambda-l}{2}+1\right)} \frac{\Gamma\left(\frac{l}{2}+1+\frac{i \sqrt{k}}{2}\right) \Gamma\left(\frac{l}{2}+1-\frac{i \sqrt{k}}{2}\right)}{\sqrt{k} \Gamma\left(\frac{l+1}{2}+\frac{i \sqrt{k}}{2}\right) \Gamma\left(\frac{l+1}{2}-\frac{i \sqrt{k}}{2}\right)}} ;
\end{aligned}
$$

here we have used Euler's gamma-function $\Gamma$. The inverse of (3.18) is clearly $X^{a}=[g(\lambda)]^{-1} \bar{x}^{a}[g(\lambda)]^{-1}$. We have thus explicitly constructed a $*$-algebra isomorphism

$$
\mathscr{A}_{\Lambda}:=\operatorname{End}\left(\mathscr{H}_{\Lambda}\right) \simeq M_{N}(\mathbb{C}) \simeq \pi_{\Lambda}[U \operatorname{so}(4)], \quad N:=(\Lambda+1)^{2} .
$$




\section{$3.2 *$-Automorphisms of the algebra of observables}

Within the group of $*$-automorphisms of the algebra of observables $M_{N}(\mathbb{C}) \simeq \mathscr{A}_{\Lambda}$

$$
b \rightarrow g b g^{-1}, \quad b \in \mathscr{A}_{\Lambda}, \quad g \in S U(N)
$$

again a special role is played by the subgroup $S O(4)$ acting through the representation $\pi_{\Lambda}$, namely $g=\boldsymbol{\pi}_{\Lambda}\left[e^{i \alpha}\right], \alpha \in \operatorname{so}(4) . O(3) \subset S O(4)$ plays the role of isometry subgroup. In particular, choosing $\alpha=\alpha_{i} L_{i}\left(\alpha_{i} \in \mathbb{R}\right)$ the automorphism amounts to a $S O(3)$ transformation (a rotation in $\mathbb{R}^{3}$ ). An $O(3)$ transformation with determinant -1 in the $X^{1} X^{2} X^{3}$ space, and therefore also in the $\bar{x}^{1} \bar{x}^{2} \bar{x}^{3}$ space, is parity: $\left(L_{i}, X^{i}\right) \mapsto\left(L_{i},-X^{i}\right)$, or equivalently $E_{i}^{1} \leftrightarrow E_{i}^{2}$, the only automorphism of $s o(4)$ (corresponding to the exchange of the two nodes in the Dynkin diagram).

\subsection{Convergence to $O(3)$-covariant quantum mechanics on $S^{2}$ as $\Lambda \rightarrow \infty$}

Define the $O(3)$-covariant embedding $\mathscr{I}: \mathscr{H}_{\Lambda} \hookrightarrow \mathscr{L}^{2}\left(S^{2}\right) \equiv \mathscr{H}_{s}$ by setting $\mathscr{I}\left(\psi_{l}^{m}\right):=Y_{l}^{m}$ and applying linear extension; below we drop $\mathscr{I}$ and identify $\psi_{l}^{m}=Y_{l}^{m}$ as elements of the Hilbert space. Clearly $P_{\Lambda} \phi \rightarrow \phi$ in the $\mathscr{H}_{s}$-norm \|\| , for all $\phi \in \mathscr{H}_{s} ; \mathscr{H}_{\Lambda}$ 'invades' $\mathscr{H}_{s}$ as $\Lambda \rightarrow \infty$.

$\mathscr{I}$ induces the embedding of operator algebras $\mathscr{J}: \mathscr{A}_{\Lambda} \hookrightarrow B\left[\mathscr{H}_{s}\right]$, with $\mathscr{A}_{\Lambda}$ annihilating $\mathscr{H}_{\Lambda}^{\perp}$; $\overline{L_{i}}=L_{i}$ on $\mathscr{H}_{\Lambda}$, and $\bar{L}_{i} \phi \rightarrow L_{i} \phi$ as $\Lambda \rightarrow \infty$, for all $\phi \in D\left(L_{i}\right) \subset \mathscr{H}_{s}$. Bounded (resp. continuous) functions $f$ on $S^{2}$, acting as multiplication operators $f \cdot: \phi \in \mathscr{H}_{s} \mapsto f \phi \in \mathscr{H}_{s}$, make up a subalgebra $B\left(S^{2}\right)$ [resp. $\left.C\left(S^{2}\right)\right]$ of $B\left[\mathscr{H}_{S}\right]$. We define the fuzzy analog of the vector space $B\left(S^{2}\right)$ as

$$
\begin{gathered}
\mathscr{C}_{\Lambda}:=\left\{\sum_{l=0}^{2 \Lambda} \sum_{m=-l}^{l} f_{l}^{m} \widehat{Y}_{l}^{m}, f_{l}^{m} \in \mathbb{C}\right\}, \\
\text { where } \quad \widehat{Y}_{l}^{m}:=M_{l} \sqrt{\frac{(l+m) ! 2^{l-m}}{(2 l) !(l-m) !}} L_{-}^{l-m}\left(\bar{x}^{+}\right)^{l}
\end{gathered}
$$

are the fuzzy analogs of $Y_{l}^{m} \cdot \in B\left(S^{2}\right) . \mathscr{C}_{\Lambda} \subset \mathscr{A}_{\Lambda}$ as a vector space, but not as a subalgebra. The decomposition of $\mathscr{C}_{\Lambda}$ in irreducible representations of $O(3)$ reads $\mathscr{C}_{\Lambda}=\bigoplus_{l=0}^{2 \Lambda} V_{l}$. In [1] we have shown that for all $\phi \in \mathscr{H}_{s} \bar{x}^{i} \phi \rightarrow\left(x^{i} / r\right) \phi$; more generally, setting $\hat{f}_{\Lambda}:=\sum_{l=0}^{2 \Lambda} \sum_{|m| \leq l} f_{l}^{m} \widehat{Y}_{l}^{m} \in \mathscr{A}_{\Lambda}$ for all $f \in B\left(S^{2}\right)$ we find

Proposition 4.3 in [1]. Choose $k(\Lambda) \geq 2^{3 \Lambda+3} \Lambda^{\Lambda+5}(\Lambda+1)$. Then $\hat{f}_{\Lambda} \rightarrow f \cdot \widehat{(f g)}{ }_{\Lambda} \rightarrow f g \cdot$, $\hat{f}_{\Lambda} \hat{g}_{\Lambda} \rightarrow f g$. strongly as $\Lambda \rightarrow \infty, \forall f, g \in B\left(S^{2}\right)$.

On the other hand, the corresponding convergences in the operator norm do not hold, because for all $\Lambda>0$ the operators $\bar{x}^{i}, \bar{L}_{i}$ annihilate $\mathscr{H}_{\Lambda}^{\perp}$, whereas $x^{i} / r, L_{i}$ do not.

\section{Comparison with the literature, final remarks and outlook}

In conclusion, for $d=1,2$ we have built a sequence $\left(\mathscr{A}_{\Lambda}, \mathscr{H}_{\Lambda}\right)$ of finite-dim, $O(D)$-covariant $(D=d+1)$ approximations of quantum mechanics of a spinless particle on the sphere $S^{d} ; \mathscr{R}^{2} \gtrsim 1$ collapses to 1 as $\Lambda \rightarrow \infty$. This result has been achieved imposing an energy-cutoff $E \leq \Lambda(\Lambda+d-1)$ on quantum mechanics of a particle in $\mathbb{R}^{D}$ subject to a sharp confining potential $V(r)$ on the sphere $r=1 . \mathscr{A}_{\Lambda}$ is a fuzzy approximation of the whole algebra of observables of the particle on $S^{d}$ 
(phase space algebra), and converges to it in the limit $\Lambda \rightarrow \infty$. We have explicitly determined a $*-$ isomorphism $\mathscr{A}_{\Lambda} \simeq \pi_{\Lambda}[U s o(D+1)]$, with a suitable irreducible representation $\pi_{\Lambda}$ of $U s o(D+1)$ on $\mathscr{H}_{\Lambda}$. On the other hand $\mathscr{H}_{\Lambda}$ carries a reducible representation of the $U s o(D)$ subalgebra generated by the $\bar{L}_{i j}: \quad \mathscr{H}_{\Lambda}$ is the direct sum of all irreducible representations fulfilling $L^{2} \leq \Lambda(\Lambda+d-1)$. A similar decomposition holds for the subspace $\mathscr{C}_{\Lambda} \subset \mathscr{A}_{\Lambda}$ of completely symmetrized polynomials in the $\bar{x}^{i}$ acting as multiplication operators on $\mathscr{H}_{\Lambda}$. As $\Lambda \rightarrow \infty$ these respectively become the decompositions (1.2) of $\mathscr{L}^{2}\left(S^{d}\right)$ and of $C\left(S^{d}\right)$ acting on $\mathscr{L}^{2}\left(S^{d}\right)$.

Our approach seems applicable to $d \geq 3$; this will allow a more direct comparison with the rest of the literature. The fuzzy spheres of dimension $d=4$ introduced in [18], as well as the $d \geq 3$ ones considered in $[19,20,21]$, are based on $\operatorname{End}(V)$, where $V$ carries a particular irreducible representation of $S O(D)$; as $\mathscr{R}^{2}$ is central, it can be set $\mathscr{R}^{2}=1$ identically. The commutation relations are also Snyder-like, hence $O(D)$-covariant. The fuzzy spherical harmonics are elements of $\operatorname{End}(V)$, but do do not close a subalgebra of $\operatorname{End}(V)$, i.e. the product $Y \cdot Y^{\prime}$ of two spherical harmonics is not a combination of spherical harmonics. This is exactly as in our models, i.e. $\mathscr{C}_{\Lambda}$ is a subspace, but not a subalgebra, of $\mathscr{A}_{\Lambda}$. (One can introduce a product in $\mathscr{C}_{\Lambda}$ by projecting the result of $Y \cdot Y^{\prime}$ to the vector space $\mathscr{C}_{\Lambda}$, but it will be non-associative).

In $[22,23]$ Steinacker and Sperling consider the possibility of construncting a fuzzy 4-sphere $S_{N}^{4}$ through a reducible representation of $U s o(5)$ on a Hilbert space $V$ obtained decomposing an irreducible representation $\pi$ of $U s o(6)$ characterized by a triple of highest weights $\left(N, n_{1}, n_{2}\right)$; so $\operatorname{End}(V) \simeq \pi[U s o(6)]$, in analogy with our result. The elements $X^{i}$ of a basis of the vector space $s o(6) \backslash s o(5)$ play the role of noncommuting cartesian coordinates. Hence, the $O(5)$-scalar $\mathscr{R}^{2}=$ $X^{i} X^{i}$ is no longer central, but its spectrum is still very close to 1 provided $N \gg n_{1}, n_{2}$, because then $V$ decomposes only in few irreducible $S O(5)$-components, all with eigenvalues of $\mathscr{R}^{2}$ very close to 1 ; if $n_{1}=n_{2}=0$ then $\mathscr{R}^{2} \equiv 1(V$ carries an irreducible representation of $O(5))$, and one recovers the fuzzy 4-sphere of [18]. On the contrary, in our approach $\mathscr{R}^{2} \simeq 1$ is guaranteed by adopting $\bar{x}^{i}=g\left(L^{2}\right) X^{i} g\left(L^{2}\right)$ rather than $X^{i}$ as noncommutative cartesian coordinates, and $\mathscr{R}^{2}=\bar{x}^{i} \bar{x}^{i}$.

Many other aspects of these new fuzzy spheres deserve investigations: e.g. space uncertainties, optimally localized states (coherent states [24]), their distance (as done e.g. in [25] for the FS), extension to particles with spin, etc. We hope that progresses on these and other issues can be reported soon.

\section{Acknowledgments}

We are grateful to F. D'Andrea and T. Weber for useful discussions. This article is based upon work from COST Action MP1405 QSPACE (Quantum Structure of Spacetime), supported by COST (European Cooperation in Science and Technology).

\section{References}

[1] G. Fiore, F. Pisacane, J. Geom. Phys. (2018), DOI: 10.1016/j.geomphys.2018.07.001. arXiv:1709.04807.

[2] H. S. Snyder, Phys. Rev. 71 (1947), 38.

[3] C. A. Mead, Phys. Rev. 135 (1964), B849. 
[4] S. Doplicher, K. Fredenhagen, J. E. Roberts, Phys. Lett. B 331 (1994), 39-44; Commun. Math. Phys. 172 (1995), 187-220;

[5] A. Connes and J. Lott, Nucl. Phys. B (Proc. Suppl.) 18 (1990) 29

[6] A. H. Chamseddine and A. Connes, Fortsch. Phys. 58 (2010) 553; and references therein.

[7] A. Connes, Noncommutative geometry, Academic Press, 1995.

[8] J. Madore, An introduction to noncommutative differential geometry and its physical applications, Cambridge University Press, 1999.

[9] J. M. Gracia-Bondia, H. Figueroa, J. Varilly, Elements of Non-commutative geometry, Birkhauser, 2000.

[10] G. Landi, Giovanni (1997), An introduction to noncommutative spaces and their geometries, Lecture Notes in Physics 51, Springer-Verlag, 1997

[11] J. Madore, Journ. Math. Phys. 32 (1991) 332; Class. Quantum Grav. 9 (1992), 6947.

[12] J. Hoppe, Quantum theory of a massless relativistic surface and a two-dimensional bound state problem, PhD thesis, MIT 1982; B. de Wit, J. Hoppe, H. Nicolai, Nucl. Phys. B305 (1988), 545.

[13] H. Grosse, J. Madore, Phys. Lett. B283 (1992), 218.

[14] H. Grosse, C. Klimcik, P. Presnajder, Int. J. Theor. Phys. 35 (1996), 231-244.

[15] P. Aschieri, H. Steinacker, J. Madore, P. Manousselis, G. Zoupanos SFIN A1 (2007) 25-42; and references therein.

[16] A. Y. Alekseev, A. Recknagel, V. Schomerus, JHEP 09 (1999) 023.

[17] Y. Hikida, M. Nozaki and Y. Sugawara, Nucl. Phys. B617 (2001), 117.

[18] H. Grosse, C. Klimcik, P. Presnajder, Commun. Math. Phys. 180 (1996), 429-438.

[19] S. Ramgoolam, Nucl. Phys. B610 (2001), 461-488; JHEP 0210 (2002) 064; and references therein.

[20] B. P. Dolan and D. O’Connor, JHEP 0310 (2003) 06.

[21] B. P. Dolan, D. O’Connor and P. Presnajder, JHEP 0402 (2004) 055.

[22] H. Steinacker, J. High Energy Physics 2016: 156.

[23] M. Sperling, H. Steinacker, J. Phys. A: Math. Theor. 50 (2017), 375202.

[24] A. Perelomov, Generalized Coherent States and Their Applications, Springer-Verlag, 1986.

[25] F. D’Andrea, F. Lizzi, P. Martinetti, J. Geom. Phys. 82 (2014), 18-45. 